\title{
DESARROLLO DE INGENIERÍA PARA DESCARGAR EL CLINKER EN EL NUEVO GALPON
}

\section{DEVELOPMENT OF ENGINEERING TO DOWNLOAD THE CLINKER IN THE NEW GALPON}

\author{
PhD. Luis Orlando Cotaquispe Zevallos*. \\ * Pontificia Universidad Católica del Perú, Facultad de Ciencias e Ingeniería. \\ Av. Universitaria 1801, Lima, Lima, Perú. \\ Teléfono: (511) 6262000. \\ E-mail: lcotaqu@ pucp.edu.pe
}

Resumen: El alcance del proyecto para el transporte y descarga del Clinker al nuevo Galpón en la planta de Otavalo - Quito de UNACEM - Ecuador, se logró optimizando el diseño relacionado con el circuito del Clinker a través de una faja transportadora de 36", una torre estructural vertical porta ducto de descarga y la cimentación de la Torre (ver figura). Por lo tanto, se diseñó el circuito óptimo para un traslado seguro y eficiente para las cargas actuantes sobre los diversos equipos, en particular sobre la celosía porta faja transportadora del circuito que se observa en la figura; además la ubicación de los soportes de apoyo y su distribución en la torre de concreto han sido críticos para el análisis por rigidez de las estructuras portantes de estos accesorios. Resulta clave señalar que para este proyecto el tema de las cargas actuantes por equipo, fue determinante para el diseño de la celosía y sus soportes, así como de la torre vertical.

Palabras clave: Diseño, carga, faja transportadora y estructuras

Abstract: The scope of the project for the transport and discharge of Clinker to the new
Warehouse at the Otavalo - Quito plant of UNACEM - Ecuador, was achieved by
optimizing the design related to the Clinker circuit through a 36 "conveyor belt, a
structural tower vertical discharge duct and the foundation of the Tower (see figure).
Therefore, the optimal circuit was designed for a safe and efficient transfer for the loads
acting on the various equipment, in particular on the lattice of the conveyor belt of the
circuit shown in the figure; In addition, the location of the support supports and their
distribution in the concrete tower have been critical for the rigidity analysis of the
supporting structures of these accessories. It is key to point out that for this project the
issue of loads acting on equipment was decisive for the design of the lattice and its
supports as well as the vertical tower.

Keywords: Design, load, belt transporter and structures. 


\section{INTRODUCCIÓN}

En el desarrollo de la Ingeniería de Diseño resulta importante definir los procesos generales de funcionamiento del proyecto referidos a la Faja transportadora, a la Torre vertical y al Cajón de descarga colocado por la parte de afuera de la Torre, con lo cual se pueden determinar las cargas actuantes sobre las estructuras

\section{DATOS DEL PROYECTO}

Luego de analizar la información recibida, así como los planos referentes a la zona en donde se desarrollará el proyecto se procedió a realizar la proyección acerca del volumen del trabajo solicitado con la finalidad de definir el alcance del proyecto del transporte y descarga del Clinker al nuevo Galpón en la planta de Otavalo - Quito de UNACEM - Ecuador, relacionado con el análisis y cálculo estructural de la celosía para la faja transportadora de 36 ", Torre vertical porta ducto de descarga y la cimentación de la Torre (ver figura 1)

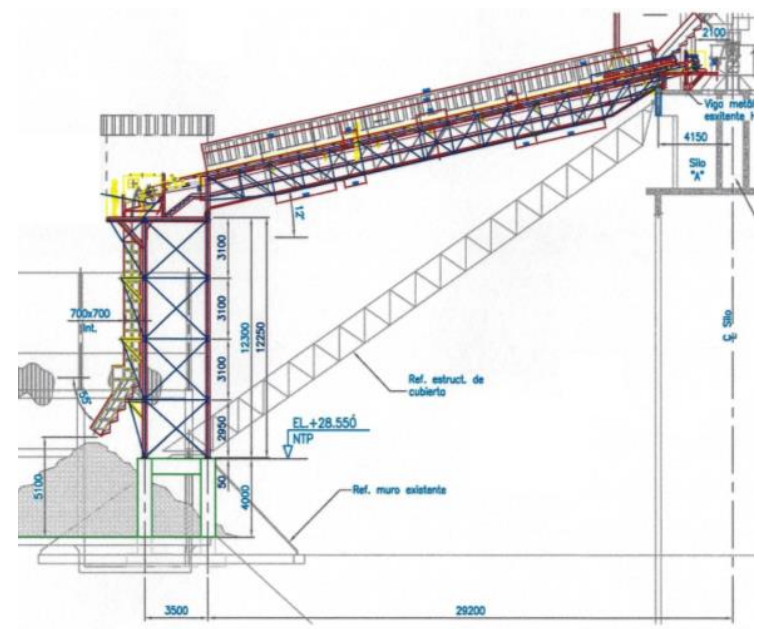

Fig. 1. Diseño final del circuito de transporte Clinker al nuevo Galpón

Resulta claro que, para poder entender el circuito del tránsito óptimo presentado por el equipo de diseño y aprobado por los responsables de la planta, se requiere además de una serie de componentes necesarios y complementarios para garantizar el flujo del transporte del Clinker al nuevo Galpón.

\subsection{Faja Transportadora}

En la Tabla 1 se presentan los resultados del análisis del sistema de cargas en el plano de elevación $X-Z$ de la celosía de apoyo para la faja.
Tabla 1: Datos de la Faja de 30"

\begin{tabular}{|c|c|c|c|c|c|c|}
\hline Equipo & $\begin{array}{c}\text { Tipo de } \\
\text { Bastidor }\end{array}$ & $\begin{array}{c}B \\
(\mathrm{~mm})\end{array}$ & $\begin{array}{c}\text { Angulo } \\
(\beta)\end{array}$ & $\begin{array}{c}L \\
(\mathrm{~m})\end{array}$ & $\begin{array}{c}R_{2} \\
(\mathrm{Ton})\end{array}$ & $\begin{array}{c}R_{\text {silo }} \\
(\text { Ton })\end{array}$ \\
\hline Faja 30" & Celosía & 760 & $-12^{\circ}$ & 25.7 & 12.28 & 9.60 \\
\hline
\end{tabular}

\subsection{Torre vertical}

A continuación, se presenta el resultado en función de las cargas actuantes por niveles sobre la Torre vertical:

- Carga vertical en la torre por la celosía:
$(12280 / 2) \mathrm{kg}$
$(\downarrow)$ nivel N: $+40.85 \mathrm{~m}$.

- Carga sistema templador + polea cola:
$(1630 / 4) \mathrm{kg}$
$(\downarrow)$ nivel $\mathrm{N}:+40.85 \mathrm{~m}$.

- Carga viva + pasarela:
(570)
$\mathrm{kg}$
$(\downarrow)$ nivel N: $+40.85 \mathrm{~m}$.

- Carga del cajón vertical para descarga:
(6500/4) kg
( $\downarrow$ ) nivel N: +40.85 m.

\section{DISEÑO DE LA CELOSÍA ESTRUCTURAL DE LA FAJA}

La faja transportadora de $30^{\prime \prime}$ es la que trabaja con una inclinación de $-12^{\circ}$ en la salida de la zona de descarga al ducto. En base a este funcionamiento se han evaluados y seleccionados todas las cargas que actúan sobre la celosía diseñada (ver figura 2).

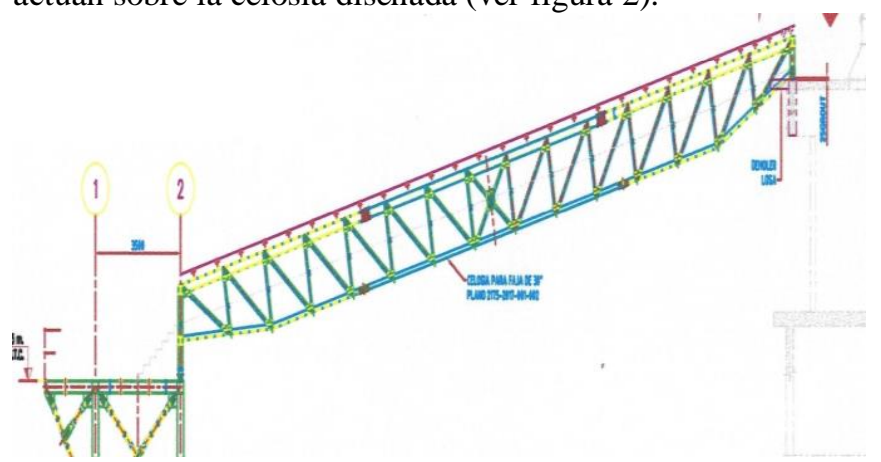

Fig. 2. Diseño estructural óptimo de la celosía

\subsection{Diseño y cálculo de la Celosía}

El estado desfavorable de las cargas distribuida y puntual sobre la celosía (Hibbeler, 1997) ocurre por la inclinación entre los apoyos \#2 de la Torre vertical y el apoyo para la zona del silo de concreto en donde ocurre el ingreso del Clinker. Entonces se evalúa el modelo presentado para ese caso y se obtiene finalmente las reacciones, momentos flectores y la zona crítica de la estructura denominada celosía (ver figura 2). 
El diseño y análisis estructural se desarrolló con el programa de cálculo estructural (SAP 2000, V.17), para la forma constructiva típica de la celosía con 2 canales [ ] en la zona superior y con dos perfiles angulares $L\lrcorner$ en la zona inferior (ver figura 3 ).

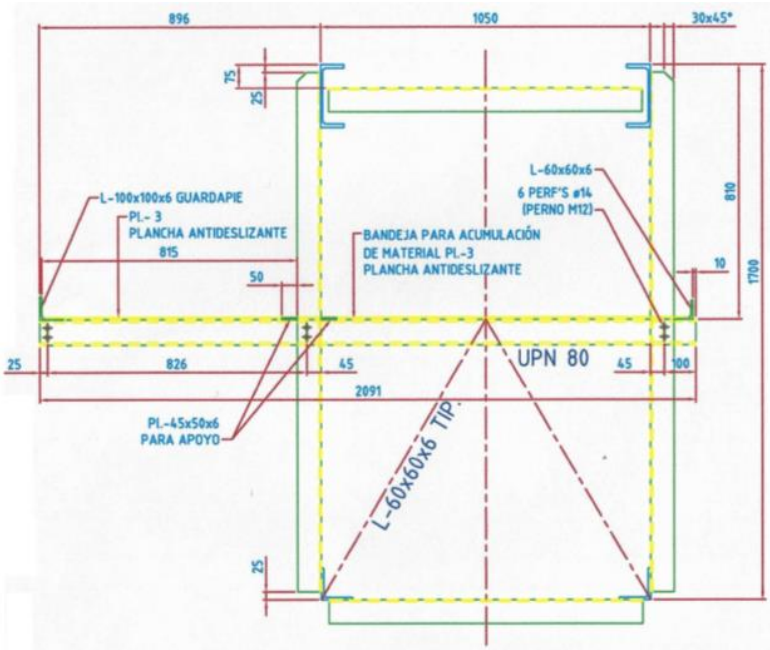

Fig. 3. Sección crítica de los componentes de la celosía estructural

Además, se evalúan los diagramas de fuerzas cortantes y del momento flector en conjunto con la finalidad de reconocer la zona más solicitada, la cual resulto estar entre los apoyos \#2 y el silo (zona central). Esta zona se evalúa por resistencia y deformación para el plano X - Z; con la finalidad de verificar el factor de seguridad y la rigidez recomendadas por la Norma Internacional ANSI (AISC-360, 2005) y la Norma Ecuatoriana de la Construcción (NEC-SE-AC, 2015).

En la tabla 2 se presentan los resultados del análisis del sistema de cargas combinadas en el plano X Z; considerando como sección típica de la celosía, la que se muestra en la figura 3 .

\section{Tabla 2: Estado de cargas combinado}

\begin{tabular}{|c|c|c|c|c|c|}
\hline $\begin{array}{c}\text { Tipo de } \\
\text { Bastidor }\end{array}$ & $\begin{array}{c}\text { Angulo } \\
(\beta)\end{array}$ & $\begin{array}{c}A_{T} \\
\left(\mathrm{~m}^{2}\right)\end{array}$ & $\begin{array}{c}I \\
\left(\mathrm{~m}^{4}\right)\end{array}$ & $\begin{array}{c}V \\
(\text { ton })\end{array}$ & $\begin{array}{c}M_{\text {centro }} \\
\text { (ton x m) }\end{array}$ \\
\hline Celosía & $-12^{\circ}$ & 0.043 & 0.0179 & 18331 & 235.6 \\
\hline
\end{tabular}

Entonces se tiene un sistema de cargas combinadas el cual será resuelto por la teoría de Von Mises (VM) el resultado de reemplazar los valores de la tabla 2 para el esfuerzo equivalente se muestran en la tabla 3
Tabla 3: Factor de seguridad carga combinada

\begin{tabular}{|c|c|c|c|c|c|}
\hline $\begin{array}{l}\text { Tipo de } \\
\text { Bastidor }\end{array}$ & $\begin{array}{c}T_{\text {孟 }} \\
\left(\mathrm{kg} / \mathrm{mm}^{2}\right)\end{array}$ & $\begin{array}{c}\sigma_{F} \\
\left(\mathrm{~kg} / \mathrm{mm}^{2}\right)\end{array}$ & $\begin{array}{c}\sigma_{\text {es }} \\
\left(\mathrm{kg} / \mathrm{mm}^{2}\right)\end{array}$ & $\begin{array}{c}\sigma_{p} \text { adime } \\
\left(\mathrm{kg} / \mathrm{mm}^{2}\right)\end{array}$ & $F S$ \\
\hline Celosía & 1.11 & 10.98 & 11.15 & 25.00 & 2.24 \\
\hline
\end{tabular}

Es importante señalar que para terminar de analizar esta zona central referida al plano $\mathrm{X}-\mathrm{Z}$, se deben de considerar las deformaciones originadas por la carga actuante y definir la rigidez de la celosía en el plano de estudio. Este análisis está basado en el tipo de carga evaluado anteriormente el cual ha servido para hallar la deformación crítica total sobre la estructura tipo celosía en la zona central (ver tabla 4).

Tabla 4: Rigidez de la celosía en zona central

\begin{tabular}{|c|c|c|c|c|c|c|}
\hline $\begin{array}{c}\text { Tipo de } \\
\text { Bastidor }\end{array}$ & $\begin{array}{c}L \\
(\mathrm{~mm})\end{array}$ & $\begin{array}{c}\delta_{1} \\
(\mathrm{~mm})\end{array}$ & $\begin{array}{c}\delta_{2} \\
(\mathrm{~mm})\end{array}$ & $\begin{array}{c}\delta_{\mathrm{T}} \\
(\mathrm{mm})\end{array}$ & $\mathrm{R}_{\mathrm{T}}=\delta_{\mathrm{T}} / L$ & $\begin{array}{c}\mathrm{R}_{\mathrm{T}} \\
\leq \mathrm{R}_{\mathrm{ASTM}}\end{array}$ \\
\hline Celosía & 25700 & 17.24 & 8.39 & 25.63 & 0.00099 & $\leq 0.001$ \\
\hline
\end{tabular}

\subsection{Cálculo del soporte crítico}

El soporte del apoyo \#2 forma parte del extremo de la vigueta por lo tanto debe ser evaluado a pandeo estructural para la reacción de $R_{2}$ que se muestra en la tabla 1 y en la figura 4.

Además en la tabla 5 se presenta el análisis por pandeo estructural (DIN 4114, 1990) de las dos columnas soporte de apoyo \#2; las cuales soportan una carga total de 14.5 toneladas entre los 2 apoyos.

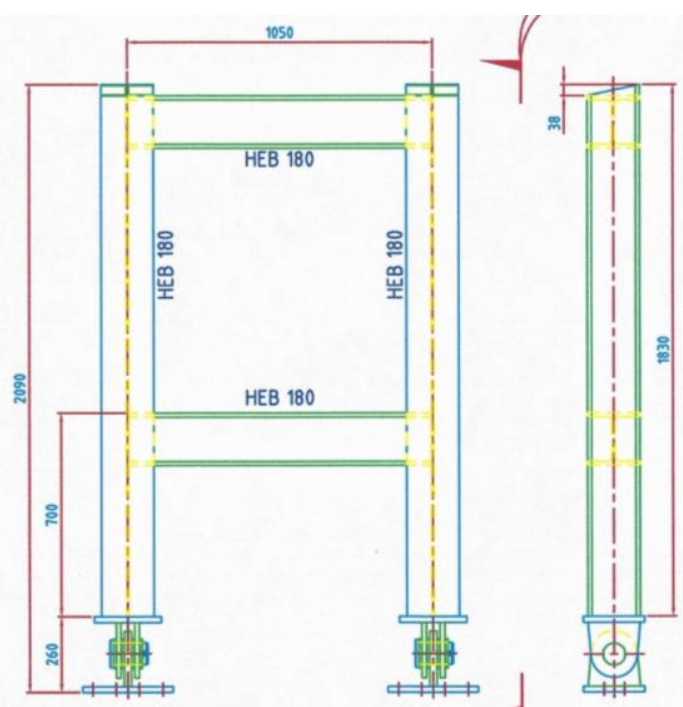

Fig. 4. Diseño estructural del soporte crítico \# 2 
Tabla 5: Cálculo por pandeo del soporte \# 2

\begin{tabular}{|c|c|c|c|c|c|c|}
\hline $\begin{array}{c}L_{P}=L \\
(\mathrm{~mm})\end{array}$ & $\lambda$ & $\omega$ & $\begin{array}{c}\sigma_{\mathrm{c}} \\
\mathrm{kg} / \mathrm{mm}^{2}\end{array}$ & $\begin{array}{c}\sigma_{\mathrm{c}} \times \omega \\
\mathrm{kg} / \mathrm{mm}^{2}\end{array}$ & $\begin{array}{c}\leqq \sigma_{\mathrm{adm}} \\
\mathrm{kg} / \mathrm{mm}^{2}\end{array}$ & $F S$ \\
\hline 2000 & 44 & 1.09 & 1.11 & 1.21 & 14 & 11.6 \\
\hline
\end{tabular}

\subsection{Cálculo del apoyo critico \# 2}

Para realizar el análisis del soporte o apoyo crítico resulta oportuno observar las figuras 5 y 6 ; las cuales son las vistas detalladas de los componentes que conforman el apoyo \#2;

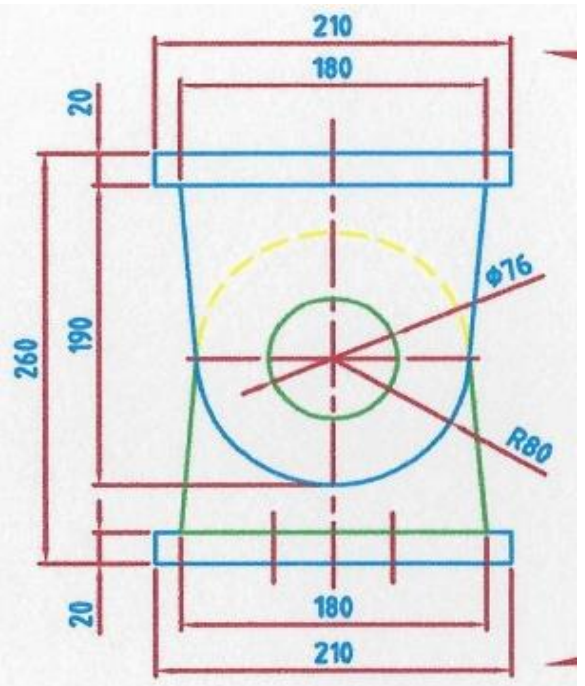

Fig. 5. Diseño del apoyo crítico \# 2

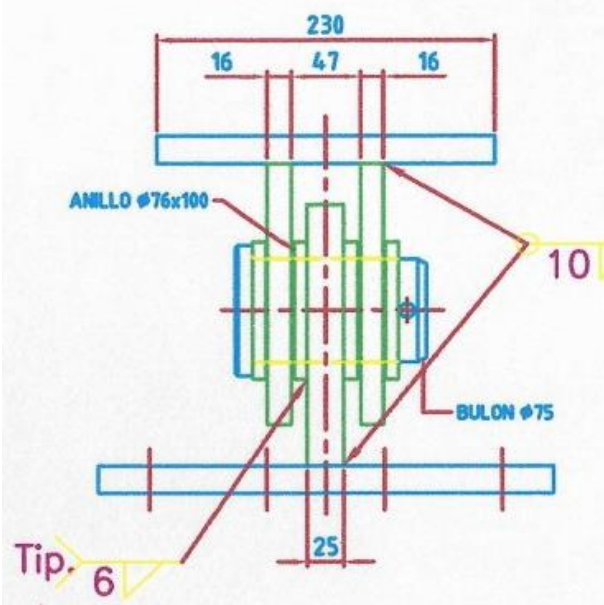

Fig. 6. Diseño de las placas del apoyo \# 2

El esfuerzo cortante que soporta el pin de conexión del apoyo \#2 debe verificarse para la carga actuante por apoyo la cual es de $F_{c}=7250 \mathrm{~kg}$, entonces para el pin de material acero CK 45 que se presenta en la figura 7 se tiene que el esfuerzo cortante será:

$$
\tau_{c}=\frac{F_{c}}{A_{c}}=\frac{7250}{4418}=1.64 \mathrm{~kg} / \mathrm{mm}^{2}
$$

Como se observa al aplicar la ecuación (1) el valor de $\tau_{c} \ll \tau_{F}=21 \mathrm{~kg} / \mathrm{mm}^{2}$ tabulado; resultó bastante menor al recomendado por la Norma DIN 17200.

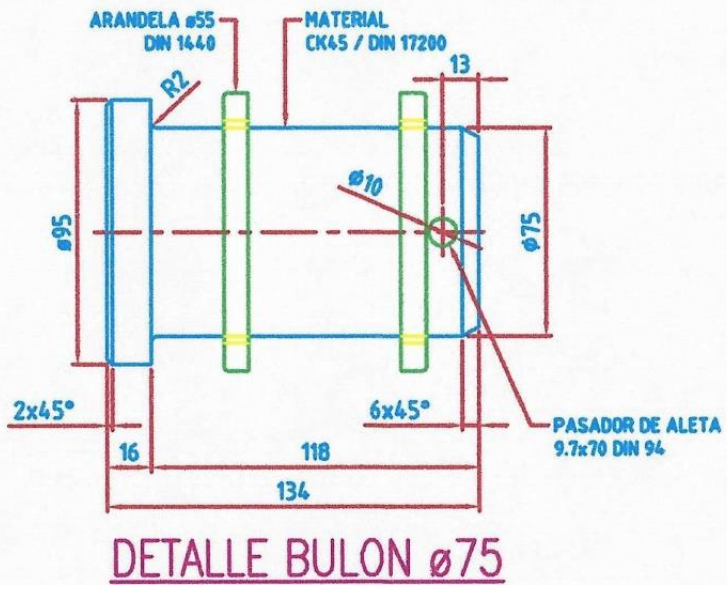

Fig. 7. Diseño del bulón o pin del apoyo \# 2

\section{DISEÑO DE LA TORRE ESTRUCTURAL PARA LA DESCARGA AL GALPÓN}

La metodología de cálculo que se aplica sobre la Torre vertical, está en función de las cargas actuantes que generan los diversos equipos que necesitan de apoyarse sobre esta torre para poder cumplir con las funciones que garanticen un óptimo funcionamiento del proceso de descarga del Clinker en el nuevo Galpón (ver figura 8).

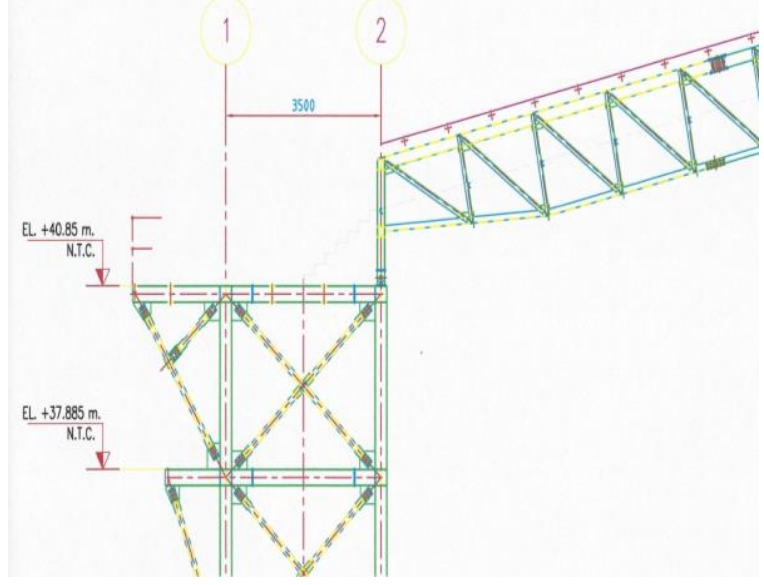

Fig. 8. Unión de la Celosía con la Torre vertical 
Resaltar que estos equipos como, el apoyo de la celosía estructural, el apoyo del cajón de descarga, pisos, barandas y las maquinarias instaladas (tambor, sistema de templado y estructura portante) en la torre vertical de transferencia están a diferentes niveles de altura. Esto implica que deberá hacerse por cada nivel un análisis de cargas más crítico, para poder garantizar un adecuado equilibrio ante las cargas externas actuantes y las cargas internas soportantes (ver figura 9). La Torre vertical pesa 8.3 ton y las columnas son del perfil resultante IPE 270 de área igual a $4590 \mathrm{~mm}^{2}$.

En general las estructuras metálicas diseñadas están unidas por tornillos acerados (VDI 2230, 1990) para facilitar su transporte y montaje de los componentes en obra.

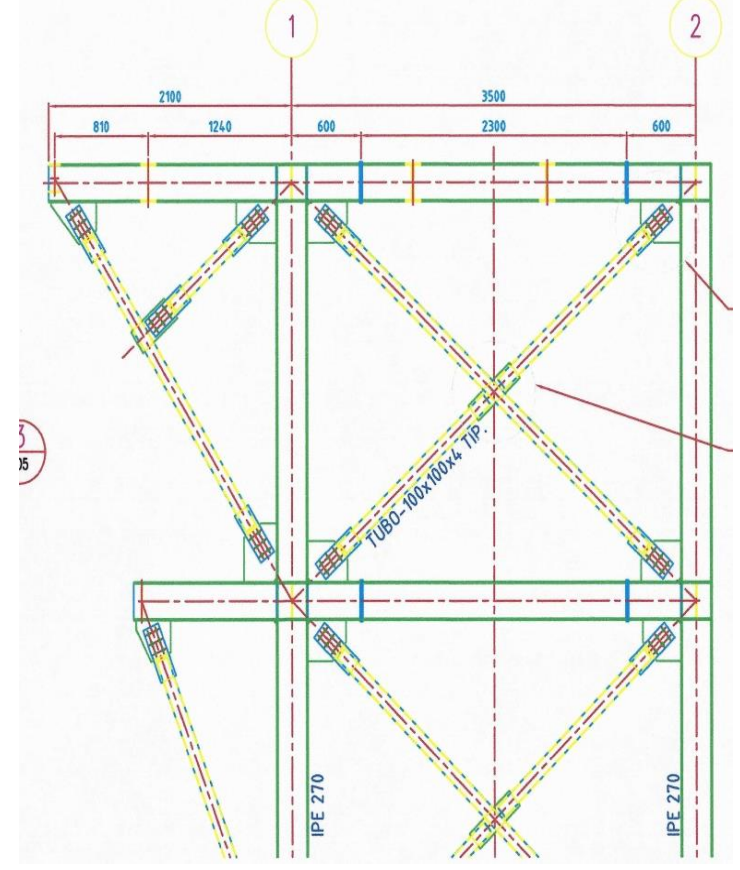

Fig. 9. Diseño de zona crítica de Torre vertical

En la tabla 6 se presenta el análisis por pandeo (método" $\omega ")$ de la columna crítica de la estructura de la torre vertical, la cual es B - 2 (ver figura 10) para el nivel $\mathrm{N}:+31.56 \mathrm{~m}$.

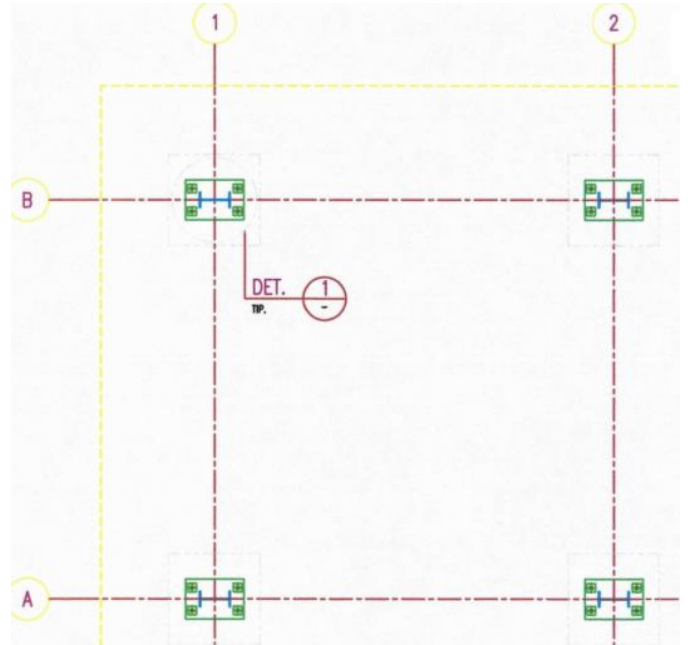

Fig. 10. Columna de apoyo crítica B-2 de la Torre vertical

Tabla 6: Cálculo por pandeo de la columna B-2

\begin{tabular}{|c|c|c|c|c|c|c|}
\hline $\begin{array}{c}L_{P}=L \\
(\mathrm{~mm})\end{array}$ & $\lambda$ & $\omega$ & $\begin{array}{c}\sigma_{\mathrm{c}} \\
\left(\mathrm{kg} / \mathrm{mm}^{2}\right)\end{array}$ & $\begin{array}{c}\sigma_{\mathrm{c}} \times \omega \\
\left(\mathrm{kg} / \mathrm{mm}^{2}\right)\end{array}$ & $\begin{array}{c}\leqq \sigma_{\mathrm{adm}} \\
\left(\mathrm{kg} / \mathrm{mm}^{2}\right)\end{array}$ & $F S$ \\
\hline 3100 & 103 & 1.79 & 1.86 & 3.33 & 14 & 4.2 \\
\hline
\end{tabular}

\subsection{Diseño de los marcos de la Torre vertical}

El DCL de los marcos para los niveles $\mathrm{N}:+40.85 \mathrm{y}$ $\mathrm{N}$ : +37.89 se presentan en las figuras 11 y 12 ; en donde se colocan las cargas críticas señaladas en la data general anterior con la finalidad de evaluar su resistencia al esfuerzo y deformación bajo cargas de trabajo. Para terminar de analizar las estructuras soportes de las plataformas en los niveles indicados se deben considerar las deformaciones originadas por las cargas actuantes y definir la rigidez de la plataforma (McCormac, 1980). Estos análisis están basados en los tipos de cargas actuantes, los cuales por el principio de superposición de efectos de las cargas servirán para evaluar las deformaciones críticas sobre la plataforma conformada por vigas tipo IPE 270. 


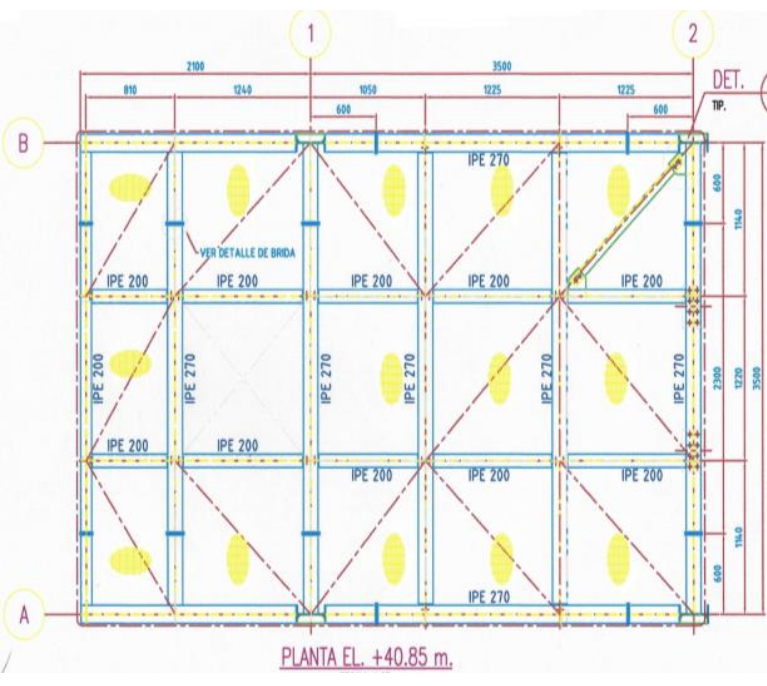

Fig. 11. Vista de plataforma en nivel $N:+40.85 \mathrm{~m}$

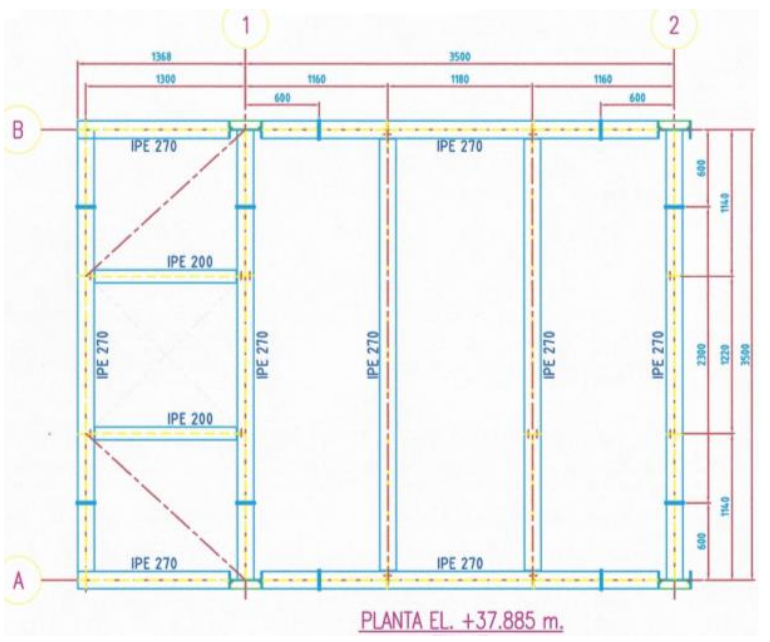

Fig. 12. Vista de plataforma en nivel $N:+37.89 m$

Como se observa de los resultados en la tabla 7, el Factor de sobrecarga $(F S)$ es confiable bajo cargas de trabajo, porque el valor de comparación es un esfuerzo admisible lo que significa que ya tiene un $F S$ incorporado en el dato, a pesar de que no se han considerado inicialmente a las estructuras de refuerzo secundarias en sus formas constructivas.

\section{Tabla 7: Análisis de esfuerzos de plataformas}

\begin{tabular}{|c|c|c|c|c|c|}
\hline $\begin{array}{c}\text { Torre } \\
\text { Vertical }\end{array}$ & Nivel & $\begin{array}{c}M \\
(\mathrm{~kg} \cdot \mathrm{m})\end{array}$ & $\begin{array}{c}\sigma_{F} \\
\left(\mathrm{~kg} / \mathrm{mm}^{2}\right)\end{array}$ & $\begin{array}{c}\sigma_{\text {F } \text { } d \mathrm{~m}} \\
\left(\mathrm{~kg} / \mathrm{mm}^{2}\right)\end{array}$ & $F S$ \\
\hline $\mathrm{B} 2-\mathrm{A} 2$ & $\mathrm{~N}:+40.85$ & 8265 & 19.27 & 25 & 1.30 \\
\hline $\mathrm{B} 1-\mathrm{A} 1$ & $\mathrm{~N}:+37.89$ & 1056.3 & 5.44 & 25 & 4.59 \\
\hline
\end{tabular}

Finalmente, en la tabla 8 se presentan los resultados del referido análisis y su comparación con la recomendación de la ASTM acerca del tema de la rigidez crítica para construcciones estructurales y cuyo valor de comparación es $\mathrm{R}_{\mathrm{ASTM}}=0.001$

Tabla 8: Análisis de rigidez de plataformas

\begin{tabular}{|c|c|c|c|c|c|}
\hline $\begin{array}{c}\text { Torre } \\
\text { Vertical }\end{array}$ & $\begin{array}{c}P \\
(\mathrm{~kg})\end{array}$ & $\begin{array}{c}\mathcal{L} \\
(\mathrm{mm})\end{array}$ & $\begin{array}{c}\delta_{1} \\
(\mathrm{~mm})\end{array}$ & $\begin{array}{c}\mathrm{R}_{\mathrm{T}}= \\
\delta_{1} / \mathrm{L}\end{array}$ & $\begin{array}{c}\mathrm{R}_{\mathrm{T}} \leq \\
\mathrm{R}_{\mathrm{ASTM}}\end{array}$ \\
\hline $\mathrm{B} 2-\mathrm{A} 2$ & 7250 & 3500 & 4.47 & 0.0013 & 0.001 \\
\hline $\mathrm{B} 1-\mathrm{A} 1$ & 812.5 & 1300 & 0.92 & 0.0007 & 0.001 \\
\hline
\end{tabular}

El escrito debe venir acompañado de un resumen en español e inglés que no supere las 150 palabras. Se recomienda que en éste se indiquen los fines del estudio o la investigación, los procedimientos básicos utilizados, los resultados más destacados y las conclusiones principales del artículo. Se deben presentar e identificar como tales entre 3 a 5 palabras claves en español y en inglés.

\section{CONCLUSIONES}

- El circuito de transporte del Clinker al nuevo Galpón presentado en el presente documento es el resultado del análisis técnico-económico de hasta tres circuitos preliminares.

- El cálculo de la celosía estructural se ha basado en la evaluación total de las cargas (vivas y muertas) que debe soportar por sostener la faja abarquillada de 30", manteniendo como una característica fundamental el tener que apoyarse en solo dos zonas. Es importante señalar que la forma constructiva de la Celosía estructural es dependiente de los componentes que la conforman y que por lo tanto su cálculo por resistencia y deformación ha sido clave para la seguridad, por lo tanto, el Factor de sobrecarga de 2.24 y su rigidez de 0.00099 están dentro del límite para garantizar un óptimo funcionamiento.

- La conexión de la Celosía estructural con la Torre vertical en el apoyo $\boldsymbol{R}_{2}$ resulta relevante para el análisis de la Torre vertical para la descarga del Clinker. Además, la Torre vertical deberá de soportar el ducto cuadrado de descarga de 6.5 ton en su periferia exterior, lo cual facilita un adecuado mantenimiento, pero esta ubicación es crítica para el diseño. 
- El ducto cuadrado de descarga del Clinker se apoya en los 4 marcos de vigas IPE270 e IPE200 soportados por las cuatro plataformas de la Torre vertical los cuales son evaluados por resistencia y deformación, obteniéndose resultados confiables para los marcos o plataformas en las tablas 7 y 8 .

- La Torre vertical soporte de apoyo de la faja abarquillada de 30 " tiene un nivel crítico en donde se concentran todas las cargas señaladas (plataforma en $\mathrm{N}:+40.85 \mathrm{~m}$ ) y además está el sistema estructural del tambor de cola de la faja, pisos de inspección y barandas. Las 4 columnas estructurales evaluadas para la carga total resultaron ser de IPE 270 y están unidas a tope con doble placa + tornillos (tipo modular) con la finalidad de facilitar su construcción y traslado.

- La columna por pandeo crítica resulto ser la B2, pero el perfil seleccionado IPE 270 garantiza un factor de sobrecarga de 4.2 a compresión.

\section{RECONOCIMIENTO}

Es importante señalar el apoyo de las empresas UNACEM PERÚ y UNACEM ECUADOR en lo referente a la data de suelos en la zona de instalación del proyecto, así mismo la prontitud acerca de señalar adecuadamente los principios fijos del diseño para que el proyecto se desarrolle sin atrasos.

\section{REFERENCIAS}

Alvernia Acevedo, S., \& Rico Bautista, D. (2017). Análisis de una red en un entorno IPV6: una mirada desde las intrusiones de red y el modelo TCP/IP. REVISTA COLOMBIANA DE TECNOLOGÍAS DE AVANZADA, $1(29)$.

C. J. Torres, J. F. Archila, M. L. Tronco, M. Becker, A. J. V. Porto, A. J. Tiberti. (2013). Estudio cinemático de una plataforma robótica para agricultura. REVISTA COLOMBIANA DE TECNOLOGÍAS DE AVANZADA, ISSN: 1692-7257. 2(22).

Hibbeler, R. (1997). Structural Analysis, Editorial Prentice Hall, Inc. USA.
Lizarazo, J. et al., (2018). Sistema de adquisición de datos para análisis de desbalance en máquinas rotativas, Colombian Journal of Advanced Technologies.Vol.1 $\quad \mathrm{N}^{\circ} 31$. Colombia.

ACI-360. (2005). Building Code. Requeriments of American Institute. USA

NEC-SE-AC. (2015). Norma Ecuatoriana de la Construcción para Acero Estructural. Ecuador.

Cárdenas, C. et al., (2018). Implementación de una mesa vibratoria triaxial neumática para el análisis de estructuras y el movimiento sísmico, Colombian Journal of Advanced Technologies. Vol.2 $\mathrm{N}^{\circ} 32$. Colombia.

DIN 4114. (1990). Übersetzungen von DINNormen. Beuth Verlag. Berlin.

VDI 2230. (1990). Berechnung von Struktur schrauben. Berlin.

McCormac, J. (1980). Structural Steel Design. Clemson University. USA.

S. A. A. Acevedo, D. R. Bautista. (2017). Análisis de una red en un entorno IPV6: una mirada desde las intrusiones de red y el modelo TCP/IP. REVISTA COLOMBIANA DE TECNOLOGÍAS DE AVANZADA, ISSN: 1692-7257. 1(29).

Torres, C., Archila, J., Tronco, M., Becker, M., Viera Porto, A., \& Tiberti, A. (2013). Estudio cinemático de una plataforma robótica para agricultura. REVISTA COLOMBIANA DE TECNOLOGÍAS DE AVANZADA, 2(22).

\section{SITIO WEB}

SAP 2000. (2018). Versión 20. Interface graphycs. www.sap2000.plataformati.microgeo.cl

> Software > CSI (cálculo estructural) 